\title{
Design and assessment of binary DNA for nanopore sequencing
}

\author{
Pelin Akan ${ }^{1 *}$, Henrik Stranneheim¹, Preben Lexow², Joakim Lundeberg ${ }^{1}$ \\ From Beyond the Genome: The true gene count, human evolution and disease genomics \\ Boston, MA, USA. 11-13 October 2010
}

DNA sequencing using an array of nanometer-sized pores (nanopores) offer an exciting option for thirdgeneration sequencing, which will allow faster and cheaper sequencing with minimal sample pre-processing. When a voltage is applied through a nanopore in a conducting fluid, a slight electric current is observed, the strength of which depends on the structure of the nanopore. When a DNA molecule passed through a nanopore, with an applied voltage, the current detected through the nanopore will differ for each base due to their differential effect on the structure of nanopore. However, current nanopore platforms cannot fully differentiate between single nucleotides due to their fast passage through the nanopore. To overcome this, Meller et al. proposed a strategy where the sequence detection is fluorescence based and involves passage of predesigned short oligonucleotides, so-called binary DNAs, encoding nucleotides [1]. In the current model, there are two binary DNA sequences and their binary combination encodes the DNA bases. Two molecular beacons complementary to the binary DNAs, each carrying a different fluorophore are also required for the fluorescence detection of DNA sequences.

The genomic DNA is sheared and every 24 bases of sequence is converted to a DNA molecule consisting of corresponding binary DNAs encoding the matching bases. The binary-encoded DNA is then hybridised with molecular beacons. Once the binary-encoded DNA passes through the nanopore, the beacons are separated from the original strand and a short burst of light is emitted which is then used to determine the base information to sequence the DNA.
We have designed sequences that serve as binary DNAs and corresponding molecular beacons. They have balanced GC content, minimal secondary structure, no cross-hybridisation and no occurrence in the human genome. A software pipeline either generates random DNA sequences or parses sequences from Archaea genomes. It then filters sequences by their GC content, repeat content, complexity, self hybridisation and occurrence in the human genome. Sequences that meet the desired criteria are then paired and tested further (e.g. cross-hybridisation). One set of a successful pair of binary DNA was assessed using the Biacore instrument, which allows interaction analysis in a label-free fashion. Our results confirmed that there is no cross hybridisation between the binary DNA sequences and they have similar hybridisation kinetics. We are currently testing the performance of molecular beacons and different hybridisation conditions (e.g. salt concentration, temperature). Our aim is to sequence an actual binary converted DNA using an array of nanopores.

\footnotetext{
Author details

${ }^{1}$ School of Biotechnology, KTH, Science for Life Laboratory, Stockholm, 171 65, Sweden. ${ }^{2}$ LingVitae, Oslo, 0277, Norway.

Published: 11 October 2010

Reference

1. Soni V. Gautam, Meller Amit: Progress toward ultrafast DNA sequencing using solid-state nanopores. Clinical Chemistry 2007, 53:1996-2001.

doi:10.1186/gb-2010-11-S1-P4

Cite this article as: Akan et al:: Design and assessment of binary DNA for nanopore sequencing. Genome Biology 2010 11(Suppl 1):P4.
} 